\title{
Characterization and inhibition of photorefractive optical damage of swift heavy ion irradiation waveguides in $\mathrm{LiNbO}_{3}$
}

\author{
Mariano Jubera, ${ }^{1, *}$ Angel García-Cabañes, ${ }^{1}$ Mercedes Carrascosa, ${ }^{1}$ José Olivares, ${ }^{2,3}$ and Fabian Lüedtke ${ }^{4}$ \\ ${ }^{1}$ Departamento de Física de Materiales, Universidad Autónoma de Madrid, Madrid 28049, Spain \\ ${ }^{2}$ Centro de Microanálisis de Materiales (CMAM), Universidad Autónoma de Madrid, Madrid 28049, Spain \\ ${ }^{3}$ Instituto de Optica, Consejo Superior de Investigaciones Científicas (CSIC), Serrano 121, Madrid 28016, Spain \\ ${ }^{4}$ Institute of Physics, University of Bonn, Wegelerstraße 8, Bonn D-53115, Germany \\ ${ }^{*}$ Corresponding author: mariano.jubera@uam.es
}

Received July 6, 2012; revised September 4, 2012; accepted September 5, 2012;

posted September 5, 2012 (Doc. ID 172137); published October 3, 2012

\begin{abstract}
The photorefractive effect and the corresponding optical damage thresholds of novel $\mathrm{LiNbO}_{3}$ waveguides fabricated by swift ion irradiation have been investigated. TE- and TM-mode operation have been characterized, and the influence of the beam propagation length analyzed. Optical damage levels similar to those of proton-exchanged waveguides have been found. In order to reduce optical damage, the influence of temperature has been investigated. An increase of more than a factor of 100 in the optical damage threshold has been obtained by moderate heating up to $90^{\circ} \mathrm{C}$. The results are briefly discussed under the two-center model for the photorefractive effect in undoped $\mathrm{LiNbO}_{3}$, and compared with data from other types of $\mathrm{LiNbO}_{3}$ waveguides. (C) 2012 Optical Society of America
\end{abstract}

OCIS codes: $\quad 130.3730,190.5330,230.4320,230.7370$.

\section{INTRODUCTION}

Photorefractive optical damage (POD) is a main drawback for high power photonic devices based on $\mathrm{LiNbO}_{3}$ crystals and waveguides [1 $1-5]$. The photorefractive effect consists in a light-induced refractive index change $\Delta n$ arising from a cascading process that combines light-induced charge transport inside the crystal with the electro-optic effect [ $\underline{6}$ ]. It produces beam degradation during propagation and light intensity limitation effects, i.e., what is generally called POD. Thus, there has been much activity devoted to characterizing and reducing it (see, for instance, [2-5,7]). Particular attention should be paid to waveguide configurations, because the long propagation lengths and the high intensities reached increase optical damage effects. In fact, a large number of works are devoted to this subject, although the data from different authors often show a considerable variability and even some contradictions (see [2] and references therein). Most experiments have been performed in Ti-indiffused and protonexchanged (PE) waveguides $[\underline{3}, \underline{8}, \underline{9}]$ whereas the information is scarcer for ion-implanted waveguides $[\underline{3}, \underline{10}]$. Regarding the theoretical description of POD in undoped $\mathrm{LiNbO}_{3}$ waveguides, a full understanding of the different manifestations of this phenomenon has been lacking for a long time. Only recently has optical damage been revised [11-13] to the light of a two-center band transport model previously proposed for photorefractive charge transport in $\mathrm{LiNbO}_{3}$ bulk crystals [14]. This theoretical approach has been able to accurately

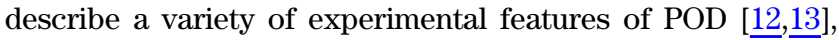
allowing for a better understanding of the phenomenon.

Over the past 10 years, a new method of producing nonlinear $\mathrm{LiNbO}_{3}$ optical waveguides using swift ion irradiation has been reported [15-19]. It involves substrate irradiation with heavy-mass ions with energies in the range $5-50 \mathrm{MeV}$, requiring much lower irradiation fluences $\left(1-4 \times 10^{14} \mathrm{~cm}^{-2}\right)$ and, thus, much shorter irradiation times in comparison with similar guides prepared by conventional ion implantation. The crystal amorphization that gives rise to the optical barrier is produced by electronic excitation processes. In other words, the method relies on the electronic energy deposition (stopping power) at variance with light ion implantation based on elastic nuclear collisions (nuclear stopping power). Moreover, due to the new amorphization mechanism of electronic instead of nuclear origin, the thickness of the optical barrier is easily programmable and can reach much higher values (up to $3-5 \mu \mathrm{m}$ ) than in the case of the usual ion implantation waveguides [19]. The guides support ordinarily and extraordinarily polarized modes and, unlike other nonlinear $\mathrm{LiNbO}_{3}$ waveguides, they show for both polarizations step-like, high-jump index profiles $\left(\Delta n_{e}=0.1, \Delta n_{o}=0.2\right)$. Initially, the guides presented moderate/high propagation losses $(1-10 \mathrm{~dB} / \mathrm{cm})[17,18]$, but very recently, using higher temperature annealing treatments $\left(350^{\circ} \mathrm{C}-375^{\circ} \mathrm{C}\right)$ and thick enough amorphization barriers, propagation losses were reduced to below $0.5 \mathrm{~dB} / \mathrm{cm}$ [20]. Furthermore, good nonlinear optical $\left(\chi_{33}\right)$ and electro-optic $\left(r_{33}\right)$ coefficients have been reported $[19,21]$, so that the novel waveguides have become good candidates for nonlinear devices. However, POD effects have been studied only preliminarily and using nonoptimized waveguides with high propagation losses of $1-5 \mathrm{~dB} / \mathrm{cm}$ [21]. Moreover, those data were taken from an $X$-cut configuration, although $Z$-cut geometries are usually preferred for efficient nonlinear applications.

Therefore, the aim of this work is to address a detailed and systematic investigation of POD of swift heavy ion irradiation 
$\mathrm{LiNbO}_{3}$ planar waveguides. The POD was studied by two complementary techniques: (i) interferometric measurement of the light-induced refractive index changes responsible for the beam damage and (ii) determination of the corresponding optical damage thresholds, i.e., maximum light intensity supported by the waveguide without distortion. In addition, we have observed the distorted output beam profile/spot to complete the information on beam degradation. TE- and TM-mode operation have been characterized and the role of the propagation length analyzed. The influence of increasing temperature is also investigated, showing that this method allows us to considerably reduce optical damage. The results are discussed in the framework of the recently reported twocenter model for POD in undoped $\mathrm{LiNbO}_{3}$ waveguides [12, $\left.\underline{13}\right]$ and compared with data from other waveguides.

\section{EXPERIMENTAL METHODS}

\section{A. Waveguide Fabrication and Basic Characterization}

1. Sample Fabrication

The waveguides have been fabricated by irradiation with fluorine ions with an energy of $30 \mathrm{MeV}$ at an incidence angle of $70^{\circ}$ and a fluence of $3 \times 10^{14} \mathrm{~cm}^{-2}$, on $Z$-cut congruently melting lithium niobate substrates purchased from Photox Optical Systems (Oxford, UK). Irradiations have been carried out in the $5 \mathrm{MV}$ tandem accelerator of the CMAM at the University Autónoma of Madrid [22]. In order to reduce propagation losses [20] after irradiation, samples have been subjected to an annealing treatment in air at $350^{\circ} \mathrm{C}$ for $90 \mathrm{~min}$.

\section{Waveguide Characterization}

The waveguides have been characterized by measuring the refractive index TE (ordinary refractive index $n_{o}$ ) and TM (extraordinary refractive index $n_{e}$ ) profiles using the prismcoupling $m$-line method with $\lambda=632.8 \mathrm{~nm}$. The fabricated waveguides support six ordinary modes and five extraordinary modes. Their profile is step-like, with a thickness of $2.4 \mu \mathrm{m}$ and refractive index jumps of 0.1 and 0.2 for extraordinary and ordinary polarization, respectively.

The propagation losses were determined through the decay of the light intensity of the guided mode at $\lambda=632.8 \mathrm{~nm}$, measured via the light scattered along the beam path recorded by a CCD camera [23]. After the annealing treatment, the waveguides present very low propagation losses below $0.5 \mathrm{~dB} / \mathrm{cm}$ for both TE and TM polarizations.

\section{B. Optical Damage Measurements}

Optical damage has been characterized using two complementary techniques: (i) interferometric measurements of the photorefractive index change and (ii) determination of the light intensity damage thresholds (IDTs) (see below).

The photorefractive index change has been measured using a Mach-Zehnder interferometer [24] recording the time evolution of the light-induced phase shift $\Delta \phi$ between the signal and reference beams until saturation. From this $\Delta \phi$ the average $|\Delta n|$ along the beam path $l$ is easily obtained as $|\Delta n|=\lambda \Delta \phi / 2 \pi l$. A schematic illustration of the setup is shown in Fig. 1(a). Optical damage is induced by a green laser beam $(\lambda=532 \mathrm{~nm}$, a wavelength very effective in generating the photorefractive effect). For the probe beam, a low intensity $633 \mathrm{~nm} \mathrm{He-Ne} \mathrm{laser} \mathrm{is} \mathrm{chosen} \mathrm{because} \mathrm{of} \mathrm{the} \mathrm{low} \mathrm{photo-}$ refractive sensitivity at this wavelength. The two laser beams

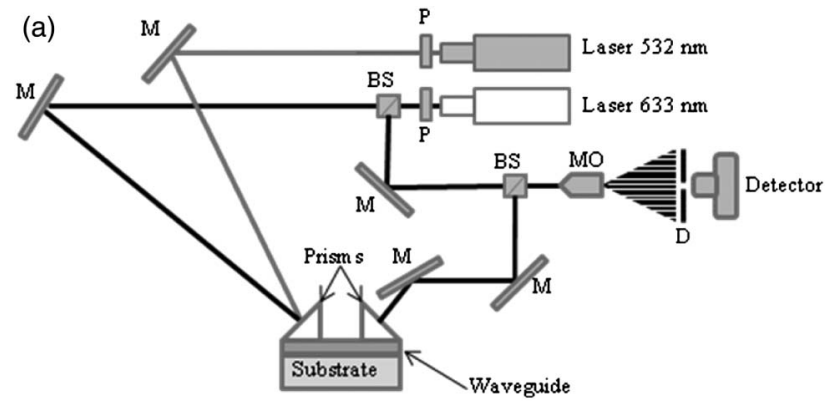

(b)

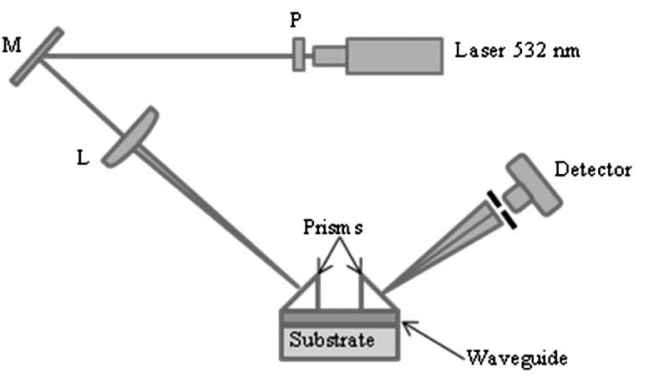

Fig. 1. (a) Schematic of the interferometric setup to measure photorefractive index changes, (b) single beam setup to determine light IDTs. P, polarizer; M, mirror; BS, beam splitter; MO, microscope objective; $\mathrm{D}$, diaphragm; $\mathrm{L}$, lens.

are coupled into the waveguide through a rutile prism, exciting the fundamental mode in both cases. When the green pump light is switched on, the effective index in the probe arm changes and a difference in the relative phase of the wavefronts is introduced. With a small diaphragm placed in front of the detector, it measures the intensity change due to movement of the light interference pattern

A standard single-beam method using incoupling and outcoupling rutile prism couplers, as described in [9] and schematically shown in Fig. 1(b), was used to determine light IDTs. The IDT is defined by the incoupled intensity $I_{\text {in }}$ at which the outcoupled intensity $I_{\text {out }}$ is no longer proportional to $I_{\text {in }}$. Then, this magnitude determines the range of intensities at which one can safely work without beam degradation along propagation. A lens is placed before the input rutile prism to facilitate reaching high incoupled intensities. A long focal length ( $300 \mathrm{~mm}$ ) is chosen so that the beam is collimated along the guide propagation length, and one single mode (the fundamental) is excited. While continuously illuminating the waveguide with light at $\lambda=532 \mathrm{~nm}$, the output power passing through a diaphragm placed $20 \mathrm{~cm}$ behind the waveguide is monitored. The size of the diaphragm is chosen such that at low intensities (no optical damage), about $70 \%$ of the total outcoupled power is transmitted. This way, the dependence $I_{\text {out }}$ versus $I_{\text {in }}$ loses linearity when the beam starts to distort.

More information about the light beam quality, particularly above the IDT, can be obtained by a profilometer that captures a digital image of the outcoupled beam.

The determination of the light intensity inside the waveguide is a key point in both experimental techniques. In all data presented in this work, we evaluate the light intensity inside the waveguide $I_{\text {in }}$ just at the input point, i.e., next to the first coupling prism, using the procedures describe in [25]. With this method a 10\%-15\% error in the determination of light intensity is estimated. 


\section{RESULTS}

A. Photorefractive Index Changes

The light-induced refractive index change $\Delta n$ experimented by the He-Ne probe beam versus the incoupled light intensity $I_{\text {in }}$ for TM $\left(n_{e}\right)$ and TE $\left(n_{o}\right)$ damage beams (fundamental modes at $\lambda=532 \mathrm{~nm}$ ) has been measured using the MachZehnder interferometer. In each case, the reading beam has the same polarization as the green pump beam and the propagation length is $7 \mathrm{~mm}$. A logarithmic plot of $|\Delta n|$ (note that $\Delta n<0$ [6] ) versus $I_{\text {in }}$ for the two polarizations is shown in Fig. 2.

In both curves one could distinguish three regions. In the first region $\left(I_{\text {in }}<40 \mathrm{~W} / \mathrm{cm}^{2}\right)$, the values of $|\Delta n|$ are nearly independent of $I_{\text {in }}$ as predicted by the standard onecenter model of the photorefractive (PR) effect [ 6 ]. From $I_{\text {in }}>40 \mathrm{~W} / \mathrm{cm}^{2},|\Delta n|$ markedly increases (region II), and finally (region III) the curves tend to saturate. Comparing the two curves in the low intensity region, $\left|\Delta n_{o}\right|$ is substantially lower than $\left|\Delta n_{e}\right|$ (by roughly a factor 3). This difference is consistent with the different magnitude of the electro-optic effect that gives rise to $|\Delta n|$. Conversely, in the high intensity region both values are unexpectedly similar, reaching a value of $|\Delta n| \sim 2.5 \times 10^{-5}$. These trends will be further discussed below in Section $\underline{4}$.

\section{B. Light Intensity Damage Thresholds}

Light IDTs have been measured for TE and TM fundamental modes for nearly the same propagation length as in Fig. 2 (in this case $l=8 \mathrm{~mm}$ ). The results are plotted in Fig. 3 . The IDT values, for which the linearity with $I_{\text {in }}$ is lost, are $\sim 50$ and $\sim 150 \mathrm{~W} / \mathrm{cm}^{2}$ for TM $\left(n_{e}\right)$ and TE $\left(n_{o}\right)$ beam propagation, respectively. Note that both IDT values are in the initial part of region II $\left|\Delta n\left(I_{\mathrm{in}}\right)\right|$, appearing at a similar value of $|\Delta n| \sim 10^{-4}$ (see Fig. 2). The lower intensity threshold for TM modes is consistent with the higher photorefractive index change exhibited in Fig. 2.

\section{Role of the Propagation Length}

As POD is a nonlinear effect, one could expect that the optical damage threshold is affected by the propagation length. However, this important aspect has rarely been considered in previous work. In fact, in some works the propagation distance of the experiments is difficult to find or simply is not specified. To investigate this point, optical damage thresholds have been measured for TE and TM modes at two additional optical lengths $l=5$ and $l=16 \mathrm{~mm}$. Figure 4 shows the obtained

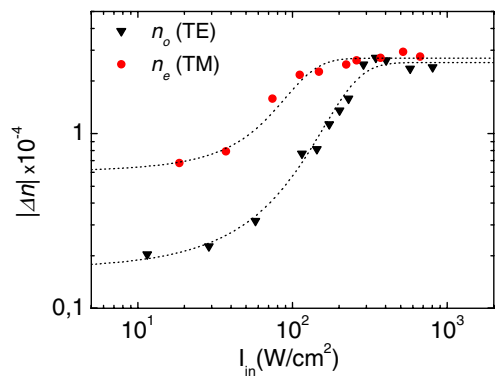

Fig. 2. (Color online) Logarithmic plot of the absolute value of the photorefractive index change $\left|\Delta n_{e}\right|$ (circles) and $\left|\Delta n_{o}\right|$ (triangles) versus the incoupled light intensity $I_{\mathrm{in}}$ inside the waveguide. (The curves are only guides to the eye.)

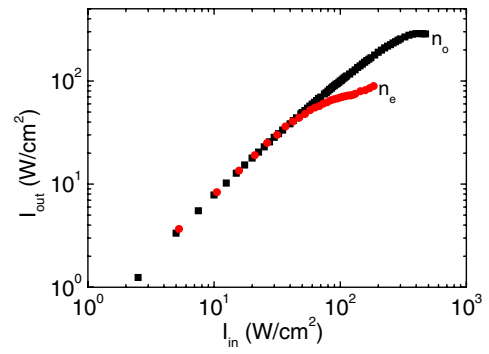

Fig. 3. (Color online) Logarithmic plot of the output intensity $I_{\text {out }}$ versus input intensity $I_{\text {in }}$ inside the waveguide for TE (squares) and TM (circles) propagating fundamental modes.

curves for TE $[\underline{4(a)}]$ and TM $[\underline{4(b)}]$. A decrease of the IDT as $l$ increases is observed. The rate between the two extreme $l$ values is about a factor of 10 for TM polarization and slightly lower for TE modes. This dependence with the propagation length can be easily understood by taking into account that for moderate and especially for high $I$, the beam undergoes photorefractive self-defocusing due to the refractive index change. This is a well known phenomenon that has been thoroughly studied in previous work on the subject (see, for instance, $[26,27])$. Therefore, the degradation of the beam is more pronounced as $l$ increases in accordance with the lower values of IDT.

\section{Beam Degradation above the Optical Damage Threshold}

In order to get more information about the degradation of the beam profile above the IDT, we have monitored the output beam profiles at a number of increasing intensities with a CCD camera. The obtained images for TM polarization $\left(n_{e}\right)$ and $8 \mathrm{~mm}$ propagation length are shown in Fig. 5 . It can be seen that above the IDT [IDT $=50 \mathrm{~W} / \mathrm{cm}^{2}$ from Fig. $\underline{4(\mathrm{~b})}$ ], the output beam starts to enlarge perpendicular to the $\overline{z \text {-axis }}$ due to a self-defocusing effect corresponding to the photorefractive decrease of $n$ in the illuminated region. For higher intensities the beam filaments show three, four, and more
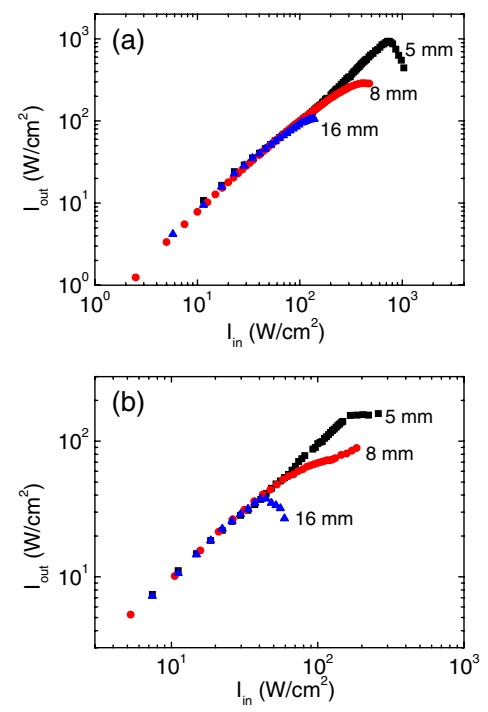

Fig. 4. (Color online) Logarithmic plot of the output intensity $I_{\text {out }}$ versus input intensity $I_{\text {in }}$ inside the waveguide for three different propagation lengths: (a) ordinary polarization (TE), (b) extraordinary polarization (TM). 


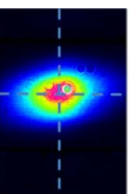

$6 \mathrm{~W} / \mathrm{cm}^{2}$

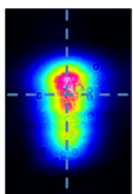

$74 \mathrm{~W} / \mathrm{cm}^{2}$

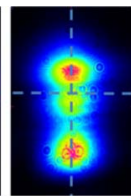

$148 \mathrm{~W} / \mathrm{cm}^{2}$

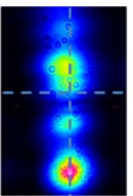

$526 \mathrm{~W} / \mathrm{cm}^{2}$

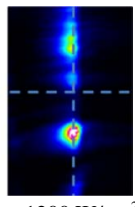

$1200 \mathrm{~W} / \mathrm{cm}^{2}$
Fig. 5. (Color online) Output beam spot images at increasing intensities for the fundamental TM mode and a propagation length $l=8 \mathrm{~mm}$. The vertical direction is parallel to the guide plane and perpendicular to the propagation direction.

spots while progressively degrading. This surprising behavior has been already observed in $Z$-cut $\alpha$-phase PE waveguides [28] and successfully explained by the two-center model [1ㅜ]

\section{E. Influence of Waveguide Temperature: Optical Damage Inhibition}

Previous results show moderate POD levels in comparison with other types of $\mathrm{LiNbO}_{3}$ waveguides, but they are still too high for efficient high power operation. Heating the sample has been reported as a method of reducing the POD in $\mathrm{LiNbO}_{3}$ crystals [29] and PE waveguides [30,31]. Furthermore, in the two-center model this reduction is well explained through the effect of the enhanced thermal excitation of electrons from $\mathrm{Nb}_{\mathrm{Li}}$ centers [12]. Thus, we have investigated whether this method also applies to swift heavy ion (SHI) waveguides. The dependence of the IDT with temperatures in the range $25^{\circ} \mathrm{C}-90^{\circ} \mathrm{C}$ has been determined in an $\mathrm{SHI} \mathrm{LiNbO}_{3}$ waveguide [see Fig. 6(a)] for extraordinary polarization. We can observe an increase of the IDT of more than a factor of 100. In fact, for moderate temperatures of $90^{\circ} \mathrm{C}$, POD appears at remarkably higher intensities of about $10 \mathrm{KW} / \mathrm{cm}^{2}$.

To further illustrate the role of temperature, we show in Fig. 6(b) how a highly distorted output beam of $I_{\text {in }}=$ $1200 \overline{\mathrm{W} / \mathrm{cm}^{2}}$ at RT (corresponding to that of the last image in Fig. 5) evolves as the temperature of the waveguide increases without changing intensity. One can clearly appreciate that beam degradation reduces following a sequence roughly the opposite of that appearing on increasing $I_{\text {in }}$ in

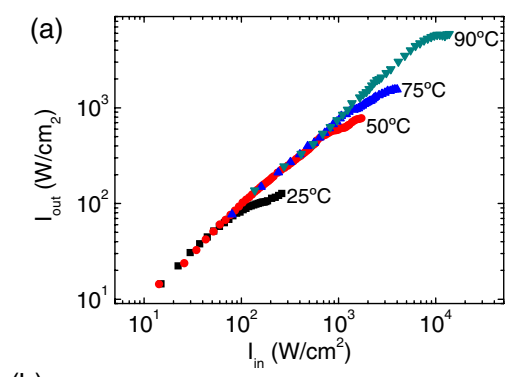

(b)

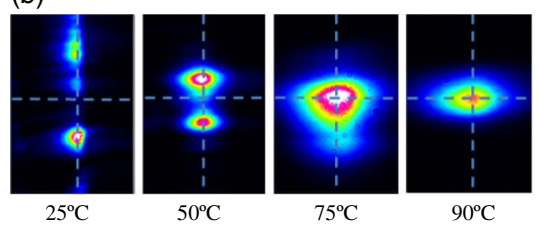

Fig. 6. (Color online) (a) Logarithmic plot of the output intensity $I_{\text {out }}$ versus input intensity $I_{\text {in }}$ inside the waveguide for TM polarization and for four different temperatures in the range $25^{\circ} \mathrm{C}-90^{\circ} \mathrm{C}(l=8 \mathrm{~mm})$ (b) corresponding output beam spot images at increasing temperatures. The vertical direction is parallel to the guide plane and perpendicular to the propagation direction.
Fig. 5. As expected, at $90^{\circ} \mathrm{C}$ a totally undistorted beam is recovered, since for this temperature the IDT appears at a higher intensity $I_{\text {in }} \sim 10000 \mathrm{~W} / \mathrm{cm}^{2}$ [see Fig. 6(a)].

\section{DISCUSSION}

The wide set of experimental data presented in this work provides an in depth characterization of $\mathrm{POD}$ in $\mathrm{SHI} \mathrm{LiNbO}_{3}$ waveguides, including photorefractive index changes, optical damage thresholds, and beam degradation profiles for intensities above the IDT. As high intensities are reached in the experiments, a first point to clarify is the possible contribution of thermo-optic effects to the refractive index change. However, since the thermo-optic coefficient of undoped $\mathrm{LiNbO}_{3}$ is positive [32], thermal refractive index changes should be also positive, leading to self-focusing instead of the observed self-defocusing behaviors. Therefore, in the range of intensities of our experiments, relevant thermo-optic contributions can be discarded.

The obtained data (both $|\Delta n|$ and IDT) for the extraordinary polarization are roughly similar to those reported for $\alpha$-phase PE waveguides [9,13,31] that only support this polarization. Note that this implies that such SHI waveguides as $\alpha$-phase PE guides [8,33] are better than Ti-indiffused and bulk crystals regarding POD. For TE modes $\left(n_{o}\right)$ the photorefractive index change (see Fig. 3) is smaller at lower and moderate intensities, although it saturates at higher intensities at roughly the same $\Delta n$ as TM modes $\left(n_{e}\right)$. To explain this nontrivial behavior, one has to consider that the electro-optic effect writes

$$
\Delta n=\frac{-1}{2} n^{3} r E
$$

$E$ being the photorefractive space charge field, $r$ the electrooptic coefficient, and $n$ the average refractive index for each polarization. In principle, one could expect a similar photorefractive field $E$ [6] for both polarizations, because the photovoltaic effect, the main charge transport mechanism giving rise to $E$ in $\mathrm{LiNbO}_{3}$ [6] , is nearly the same for both polarizations [34]. Then, using the values of the electro-optic coefficients and refractive indexes of $\mathrm{LiNbO}_{3}$, one obtains $n_{e}^{3} r_{33} / n_{0}^{3} r_{13}=2.8$, in good accordance with the factor of $\sim 3$ observed between the two curves for low intensity (see Fig. 2). On the other hand, the saturating region at high $I$ values appears when the negative $\Delta n$ profile is high enough to induce on the beam an important self-defocusing, and thus a decay of the light intensity along propagation. In other words, saturation is produced by the POD. As can be seen in Fig. 2, this effect occurs for a value of $|\Delta n| \sim 2.5-3 \times 10^{-4}$, and, as expected, it does not depend on the beam polarization. Obviously, from Eq. (1) the space charge field $E$ responsible for this $|\Delta n|$ should be higher for ordinary polarization. A similar saturating behavior of $|\Delta n|$ has been reported for PE waveguides, but in holographic experiments [35].

The shape of the obtained dependence of $|\Delta n|$ on $I$ (see Fig. 2), already observed in $\alpha$-phase PE waveguides [24], is well explained with the two-center model for the photorefractive effect in $\mathrm{LiNbO}_{3}$ waveguides recently reported [13]. According to this model, in the low intensity region, only the Fe impurity plays a role (one-center approach) [6], but as the intensity grows, a secondary center (niobium in the 
lithium site) contributes increasingly to $|\Delta n|$ [11-13]. Thus, these data can be considered a further support for the validity of the two-center photorefractive model to describe POD in undoped $\mathrm{LiNbO}_{3}$ waveguides.

The obtained results for the dependence of IDT on the propagation length $l$ prove the key role of this geometrical parameter that should be specified in any experiment of optical damage of waveguides. This result should be general and, thus, applicable to all types of $\mathrm{LiNbO}_{3}$ guides. In fact, the large dispersion of optical damage data between some papers $[\underline{2}, \underline{8}, \underline{9}]$ might be explained, at least partially, as a consequence of the different propagation length of each experimental configuration

Finally, the 100 enhancement factor of IDT from RT to $90^{\circ} \mathrm{C}$ is an important result in order to avoid optical damage for practical applications. This effect has been previously investigated for bulk crystals [29] and waveguides [36], but IDT changes seem to be smaller (the enhancement factor is 10) for similar or even larger temperature changes. Other inhibition methods, such as substrate doping with damage resistant impurities ( $\mathrm{Mg}, \mathrm{Zn}$, etc), could also be applied to SHI waveguides, although they have not been used so far. Further work in this direction should also be relevant and may further reduce optical damage effects.

\section{SUMMARY AND CONCLUSIONS}

In summary, the photorefractive effect of $\mathrm{SHI} \mathrm{LiNbO}_{3}$ waveguides has been systematically characterized, finding an intensity response in accordance with the recently reported two-center charge transport model $[12,13]$. In addition, the light IDTs for TE and TM polarizations have been determined, obtaining values that decrease with the propagation length and that are roughly similar to those of $\mathrm{PE}$ waveguides for TM (extraordinary polarization). Finally, moderate heating $\left(90^{\circ} \mathrm{C}\right)$ allows a remarkable reduction in photorefractive damage, increasing the IDT by a factor 100 , i.e., reaching values comparable to or even better than those presented by other $\mathrm{LiNbO}_{3}$ waveguides. Therefore, the set of reported data indicate that regarding $\mathrm{POD}, \mathrm{SHI} \mathrm{LiNbO}_{3}$ waveguides are really competitive with other types of $\mathrm{LiNbO}_{3}$ guides. This property, together with other advantages, such as high refractive index profile jumps for both polarizations, opens the door for their successful application for a variety of photonic devices.

\section{ACKNOWLEDGMENTS}

This work was supported by the Ministerio de Economia y Competitividad (MINECO) under grants MAT2008-06794C03-01 and MAT2011-28379-C03-01. An Formación de Personal Investigador (FPI) fellowship is acknowledged by M. Jubera.

\section{REFERENCES}

1. L. Arizmendi, "Photonic applications of lithium niobate crystals," Phys. Stat. Sol. A 201, 253-283 (2004).

2. D. Kip and M. Wesner, "Photrefractive waveguides," in Photorefractive Materials and Their Applications I, P. Gunter and J. P. Huignard, eds. (Springer, 2006), pp. 289-316.

3. T. Volk, M. Wolecke, and N. Rubinina, "Optical damage resistance in lithium niobate," in Photorefractive Materials and Their Applications II, P. Günter and J. P. Huignard, eds. (Springer, 2007), pp. 165-203.
4. S. M. Kostritskii, "Photorefractive effect in $\mathrm{LiNbO}_{3}$-based integrated-optical circuits at wavelengths of third telecom window," Appl. Phys. B 95, 421-428, (2009).

5. M. Kösters, B. Sturman, P. Werheit, D. Haertle, and K. Buse, "Optical cleaning of congruent lithium niobate crystals," Nat. Photonics 3, 510-513 (2009).

6. F. Agulló-López, G. F. Calvo, and M. Carrascosa, "Fundamentals of photorefractive phenomena," in Photorefractive Materials and Their Applications I, P. Günter and J. P. Huignard, eds. (Springer, 2006), pp. 43-77.

7. J. R. Schwesyg, M. Falk, C. R. Phillips, D. H. Jundt, K. Buse, and M. M. Fejer, "Pyroelectrically induced photorefractive damage in magnesium-doped lithium niobate crystals," J. Opt. Soc. Am. B 28, 1973-1987 (2011).

8. A. Yamada, H. Tamada, and M. Saltoh, "Photorefractive damage in $\mathrm{LiNbO}_{3}$ thin-film optical waveguides grown by liquid phase epitaxy,” J. Appl. Phys. 76, 1776-1783 (1994).

9. O. Caballero-Calero, A. Alcázar, A. García-Cabañes, J. M. Cabrera, and M. Carrascosa, "Optical damage in $X$-cut proton exchanged $\mathrm{LiNbO}_{3}$ planar waveguides,” J. Appl. Phys. 100, 093103 (2006).

10. F. Cheng, "Photonic guiding structures in $\mathrm{LiNbO}_{3}$ crystals produced by energetic ion beam," J. Appl. Phys. 106, 081101 (2009).

11. J. Carnicero, O. Caballero, M. Carrascosa, and J. M. Cabrera, "Superlinear photovoltaic currents in $\mathrm{LiNbO}_{3}$ : analyses under the two-center model," Appl. Phys. B 79, 351-358 (2004).

12. M. Carrascosa, J. Villarroel, J. Carnicero, A. García-Cabañes, and J. M. Cabrera, "Understanding light intensity thresholds for catastrophic optical damage in $\mathrm{LiNbO}_{3}$," Opt. Express 16, 115-120 (2008).

13. J. Villarroel, J. Carnicero, F. Ludtke, M. Carrascosa, A García-Cabañes, J. M. Cabrera, A. Alcazar, and B. Ramiro "Analysis of photorefractive optical damage in lithium niobate: aplication to planar waveguides," Opt. Express 18, 20852-20861 (2010).

14. E. Jermann and J. Otten, "Light-induced charge transport in $\mathrm{LiNbO}_{3}: \mathrm{Fe}$ at high light intensities," J. Opt. Soc. Am. B 10, 2085-2092 (1993).

15. H. Hu, F. Lu, F. Chen, B. Shi, K. Wang, and D. Shen, "Monomode optical waveguide in lithium niobate formed by $\mathrm{MeV} \mathrm{Si}^{+}$ion implantation," J. Appl. Phys. 89, 5224-5226 (2001).

16. G. G. Bentini, M. Bianconi, M. Chiarini, L. Correa, C. Sada, P. Mazzoldi, N. Argiolas, M. Bazzan, and R. Guzzi, "Effect of low dose high energy $\mathrm{O}^{3+}$ implantation on refractive index and linear electro-optic properties in $x$-cut $\mathrm{LiNbO}_{3}$ : planar optical waveguides formation and characterization," J. Appl. Phys. 92, 6477-6483 (2002).

17. J. Olivares, G. García, A. García-Navarro, F. Agulló-López, O. Caballero, and A. García-Cabañes, "Generation of highconfinement step-like optical waveguides in $\mathrm{LiNbO}_{3}$ by swift heavy ion-beam irradiation,” Appl. Phys. Lett. 86, 183501 (2005).

18. J. Olivares, A. Garcia-Navarro, A. Méndez, F. Agulló-López, G. García, A. García-Cabañes, and M. Carrascosa, "Novel optical waveguides by in-depth controlled electronic damage with swift ions," Nucl. Instrum. Methods B 257, 765-770 (2007).

19. J. Olivares, A. García-Navarro, G. García, F. Agulló-López, F. Agulló-Rueda, A. García-Cabañes, and M. Carrascosa, "Buried amorphous layers by electronic excitation in ion-beam irradiated luthium niobate: structure and kinetics," J. Appl. Phys. 101, 033512 (2007).

20. M. Jubera, J. Villarroel, A. García-Cabañes, M. Carrascosa, J. Olivares, F. Agullo-López, A. Méndez, and J. B. Ramiro, "Analysis and optimization of propagation losses in $\mathrm{LiNbO}_{3}$ optical waveguides produced by swift heavy-ion irradiation," Appl. Phys. B 107, 157-162 (2012).

21. J. Villarroel, M. Carrascosa, A. García-Cabañes, O Caballero-Calero, M. Crespillo, and J. Olivares, "Photorefractive response and optical damage of lithium niobate optical waveguides produced by swift-heavy ion irradiation," Appl. Phys. B 95, 429-433 (2009).

22. http://www.cmam.uam.es.

23. Y. Okamura, S. Yoshinaka, and S. Yamamoto, "Measuring mode propagation losses of integrated optical waveguides. a simple method," Appl. Opt. 22, 3892-3894 (1983). 
24. F. Luedtke, J. Villarroel, A. García-Cabañes, K. Buse, and M. Carrascosa, "Correlation between photorefractive index changes and optical damage thresholds in $z$-cut protonexchanged-LiNbO ${ }_{3}$ waveguides," Opt. Express 17, 658-665 (2009).

25. O. Caballero, J. Carnicero, A. Alcazar, G. de la Paliza, A. García-Cabañes, M. Carrascosa, and J. M. Cabrera, "Light intensity measurements in optical waveguides using prism couplers," J. Appl. Phys. 102, 074509 (2007).

26. A. Ashkin, G. D. Boyd, J. M. Dziedzic, R. G. Smith, A. A. Ballman, J. J. Levinstein, and K. Nassau, "Optically induced refractive index inhomogeneities in $\mathrm{LiNbO}_{3}$ and $\mathrm{LiTaO}_{3}$," Appl. Phys. Lett. 9, 72-74 (1966).

27. Y. Kong, J. Wen, and H. Wang, "New doped lithium niobate crystal with high resistance to photorrefraction," Appl. Phys. Lett. 66, 280-282 (1995)

28. J. Villarroel, O. Caballero-Calero, B. Ramiro, A. Alcázar, A. García-Cabañes, and M. Carrascosa, "Photorefractive non-linear beam propagation in lithium niobate waveguides above the optical damage threshold," Opt. Mater. 33, 103-106 (2010).

29. J. Rams, A. Alcazar-de-Velasco, M. Carrascosa, J. M. Cabrera, and F. Agulló-López, "Optical damage inhibition and thresholding effects in lithium niobate above room temperature," Opt. Commun. 178, 211-216 (2000).

30. A. Ikeda, T. Oi, K. Nakayama, Y. Otsuka, and Y. Fujii, "Temperature and electric field dependences of optical damage in proton- exchanged waveguides formed on MgO-doped lithium niobate crystals,” Jpn. J. Appl. Phys. 44, 1407-1409 (2005).

31. B. Chen, J. Fonseca-Campos, W. Liang, Y. Wang, and C. Q. Xu, "Wavelength and temperature dependence of photorefractive effect in quasi-phase-matched $\mathrm{LiNbO}_{3}$ waveguides," Appl. Phys. Lett. 89, 043510 (2006).

32. L. Moretti, M. Iodice, F. G. Della Corte, and I. Rendira, "Temperature dependence of the thermo-optic coefficient of LiNbO3, from 300 to $515 \mathrm{~K}$ in the visible and infrared regions," J. Appl. Phys. 98, 036101 (2005).

33. O. Caballero, A. Alcazar, J. Herrero, J. Carnicero, C. Ong, M. Domenech, G. Lifante, A. García-Cabañes, J. M. Cabrera, and M. Carrascosa, "Comparative study of optical damage and photovoltaic currents in planar $\mathrm{LiNbO}_{3}$ waveguides," Proc. SPIE 5840, 695-702 (2005).

34. R. S. Weis and T. K. Gaylord, "Lithium niobate: summary of physical properties and crystal structure," Appl. Phys. A 37, 191-283 (1985).

35. J. Villarroel, M. Carrascosa, A. García-Cabañes, and J. M. Cabrera, "Light intensity dependence of the photorefractive holographic response and dark decay of $\alpha$-phase PE waveguides," J. Opt. A 10, 104008 (2008).

36. A. Ikeda, T. Oi, K. Nakayama, Y. Otsuka, and Y. Fujii, "Temperature and electric field dependences of optical damage in protonexchanged waveguides formed on MgO-doped lithium niobate crystals," Jpn. J. Appl. Phys. 44, 1407-1409 (2005). 